Pure and Applied Mathematics Quarterly

Volume 2, Number 2

(Special Issue: In honor of

John H. Coates, Part 2 of 2$)$

$435-463,2006$

\title{
Transcendence of Periods: The State of the Art
}

\begin{abstract}
Michel Waldschmidt
Keywords: Periods, transcendental numbers, irrationality, integrals, series, Diophantine approximation, irrationality measures, transcendence measures, measures of algebraic independence, Gamma function, Beta function, zeta function, multiple zeta values (MZV).

AMS subject classifications 11J81 11J86 11J89

Abstract: The set of real numbers and the set of complex numbers have the power of continuum. Among these numbers, those which are "interesting", which appear "naturally", which deserve our attention, form a countable set. Starting from this point of view we are interested in the periods as defined by M. Kontsevich and D. Zagier. We give the state of the art on the question of the arithmetic nature of these numbers: to decide whether a period is a rational number, an irrational algebraic number or else a transcendental number is the object of a few theorems and of many conjectures. We also consider the approximation of such numbers by rational or algebraic numbers.
\end{abstract}

\section{ACKNOWLEDGMENT}

This is an english updated version of the paper in french:

Transcendance de périodes: état des connaissances,

African Diaspora Journal of Mathematics

Advances in Mathematics, Vol. 1 (2006), No. 2.

http://arXiv.org/abs/math.NT/0502582

This material presented in this paper has been the subject of lectures given between 2003 and 2005 in Sharhood (Iran), Taipei (Taiwan), Bangalore (India), Oulu (Finland), Séoul (Korea), Mahdia (Tunisia) Besançon (France) and Heraklion (Greece).

Received September 7, 2005.

À John Coates, en témoignage d'amitié et de gratitude pour tout ce qu'il m'a appris. 


\section{INTRODUCTION}

In their paper [38] whose title is "Periods", M. Kontsevich and D. Zagier introduce the notion of periods; they give two definitions and claim that they are equivalent. They propose one conjecture, two principles and five problems. The first principle reads as follows: "Whenever you meet a new number, and have decided (or convinced yourself) that it is transcendental, try to figure out whether it is a period".

If a number is not a period, then it is a transcendental number; indeed, the set of periods is a sub-algebra of $\mathbf{C}$ over the field $\overline{\mathbf{Q}}$ of algebraic numbers, hence any algebraic number is a period. We note in passing that there is no explicit known example of a complex number which is not a period (this is Problem 3 in [38]).

The goal of this paper is to study the situation when the answer is positive: what is known on the transcendence of periods?

We also consider the quantitative related aspect of this question, in connection with the following question in [38], $\S 1.2$ before their conjecture 1: we can check easily the equality between two algebraic numbers by computing both of them numerically to sufficiently high precision and using the fact that two different solutions of algebraic equations with integer coefficients of given degree and height cannot be too close to each other (this is the so-called Liouville inequality). In the example they give, due to D. Shanks [54]:

$$
\sqrt{11+2 \sqrt{29}}+\sqrt{16-2 \sqrt{29}+2 \sqrt{55-10 \sqrt{29}}}=\sqrt{5}+\sqrt{22+2 \sqrt{5}}
$$

the difference $\gamma$ between the two sides of (1) is an algebraic number of degree $\leq 16$ over the field $\mathbf{Q}(\sqrt{5}, \sqrt{29})$, hence has degree $\leq 64$ over $\mathbf{Q}$. For each of the 64 elements $\epsilon=\left(\epsilon_{1}, \ldots, \epsilon_{6}\right) \in\{0,1\}^{6}$, set

$$
\begin{aligned}
\gamma_{\epsilon}=\epsilon_{3} \sqrt{11+2 \epsilon_{2} \sqrt{29}} & +\epsilon_{4} \sqrt{16-2 \epsilon_{2} \sqrt{29}+2 \epsilon_{5} \sqrt{55-10 \epsilon_{2} \sqrt{29}}} \\
& +\epsilon_{1} \sqrt{5}+\epsilon_{6} \sqrt{22+2 \epsilon_{1} \sqrt{5}}
\end{aligned}
$$

The number

$$
N=\prod_{\epsilon} \gamma_{\epsilon}
$$

is a rational integer. It suffices to compute its numerical value with an accuracy of one digit to check that it satisfies $-1<N<1$, hence it is zero (this is the easiest case of Liouville inequality [59] § 3.5). Hence one at least of the 64 factors $\gamma_{\epsilon}$ in the product is zero; the equality (1) easily follows.

The question raised by Kontsevich and Zagier in [38] $\S 1.2$ is whether something similar can be done for periods. One should define a notion of complexity for a 
period which would be analogous to the notion of height for an algebraic number, and then to prove a lower bound of this complexity for a nonzero period, as a substitute for Liouville inequality. One of their suggestions is to count the amount of ink or the number of $\mathrm{T}_{\mathrm{E}} \mathrm{X}$ keystrokes required to write down the considered number.

It would be interesting to know whether there exist periods which are at the same time Liouville number. A positive answer to the following question would mean that there are no such Liouville periods.

Question 2. Let $\theta$ be real period. Does there exists a constant $c(\theta)>0$ such that, for any rational number $p / q$ distinct from $\theta$ with $q \geq 2$, the lower bound

$$
\left|\theta-\frac{p}{q}\right|>\frac{1}{q^{c(\theta)}}
$$

holds?

A more ambitious goal would be to ask whether real or complex periods behave, from the point of view of Diophantine approximation by algebraic numbers, like almost all real or complex numbers $[16,59]$

Question 3. Given a transcendental period $\theta \in \mathbf{C}$, does there exists a constant $\kappa(\theta)$ such that, for any nonzero polynomial $P \in \mathbf{Z}[X]$, we have

$$
|P(\theta)| \geq H^{-\kappa(\theta) d},
$$

where $H \geq 2$ is an upper bound for the (usual) height of $P$ (maximum absolute value of the coefficients) and d its degree?

\section{Abelian integrals}

The arithmetic nature of the value of the integral of an algebraic function of one variable between algebraic (or infinite) limits is now well known, both from the qualitative and quantitative point of view, as we now explain.

2.1. Genus 0: logarithms of algebraic numbers. The main tool is Baker's Theorem on linear independence, over the field $\overline{\mathbf{Q}}$ of algebraic numbers, of logarithms of algebraic numbers. Here we shall use only the following special case.

Theorem 4. Let $\alpha_{1}, \ldots, \alpha_{n}$ be nonzero algebraic numbers, $\beta_{1}, \ldots, \beta_{n}$ algebraic numbers, and, for $1 \leq i \leq n$, let $\log \alpha_{i}$ be a complex logarithm of $\alpha_{i}$. Then the number

is either zero or transcendental.

$$
\beta_{1} \log \alpha_{1}+\cdots+\beta_{n} \log \alpha_{n}
$$

Here is a consequence of Baker's Theorem 4: 
Corollary 5. Let $P$ and $Q$ be polynomials with algebraic coefficients which satisfy $\operatorname{deg} P<\operatorname{deg} Q$ and let $\gamma$ be either a closed path, or a path whose limits are either infinite or algebraic. If the integral

$$
\int_{\gamma} \frac{P(z)}{Q(z)} d z
$$

exists, then its value is either zero or transcendental.

A well known example [55] p. 97 is

$$
\int_{0}^{1} \frac{d t}{1+t^{3}}=\frac{1}{3}\left(\log 2+\frac{\pi}{\sqrt{3}}\right) .
$$

Corollary 5 follows from Theorem 4 by solving the rational fraction $P(z) / Q(z)$ into partial fractions (see for instance [48]). As a matter of fact, Corollary 5 is equivalent to Theorem 4: it suffices to write down the logarithm of an algebraic number as a period; for the principle value of the logarithm, when $\alpha$ is not a negative real number, we have for instance

while

$$
\log \alpha=\int_{0}^{\infty} \frac{(\alpha-1) d t}{(t+1)(\alpha t+1)},
$$

$$
i \pi=2 i \int_{0}^{\infty} \frac{d t}{1+t^{2}}
$$

The known measures of linear independence of logarithms of algebraic numbers (lower bounds for linear combinations, with algebraic coefficients, of logarithms of algebraic numbers - see for instance [59]) imply that the absolute value of a nonzero integral of the form (6) is explicitly bounded from below in terms of the heights and degrees of $P$ and $Q$ as well as the heights and degrees of the algebraic numbers which are the limits of the path $\gamma$.

2.2. Genus 1: elliptic integrals. The arithmetic nature of elliptic integrals of the first or the second kind has been studied as early as 1934 [50] and then 1937 [51] by Th. Schneider. Here is Theorem 15 version III in [53].

Theorem 7. The value of any elliptic integral of the first or the second kind with algebraic coefficients between algebraic limits is either zero or else transcendental.

In particular any nonzero period of an elliptic integral of the first or the second kind with algebraic coefficients is transcendental.

Theorem 16 in [53] deals with the transcendence of the quotient of two elliptic integrals of the first kind.

A consequence, which is quoted by Schneider of his Theorem 17 in [53], can be stated: The value of an elliptic integral of the first or the second kind between 
algebraic limits is the quotient of a period by a factor which is either rational or transcendental.

From Theorem 7 one deduces the result quoted in [38] § 1.1: if a and $b$ are two positive algebraic numbers, the perimeter of the ellipse with radii a and $b$

$$
2 \int_{-b}^{b} \sqrt{1+\frac{a^{2} x^{2}}{b^{4}-b^{2} x^{2}}} d x
$$

is a transcendental number. More generally the length of any arc whose limits are points with algebraic coordinates is either zero or else transcendental.

Similar results hold for a lemniscate

$$
\left(x^{2}+y^{2}\right)^{2}=2 a^{2}\left(x^{2}-y^{2}\right)
$$

when $a$ is algebraic.

These results are proved by Schneider as consequences of his results on elliptic functions. Here is for instance version I of theorem 15 in [53]. Let $\wp$ be a Weierstrass elliptic function with algebraic invariants $g_{2}$ and $g_{3}$ :

$$
\wp^{\prime 2}=4 \wp^{3}-g_{2} \wp-g_{3}
$$

Let $\zeta$ be the associated Weierstrass zeta function, $a$ and $b$ two algebraic numbers and $u$ a complex number which is not a pole of $\wp$. Assume $(a, b) \neq(0,0)$. Then one at least of the two numbers $\wp(u), a u+b \zeta(u)$ is transcendental.

Also, if one considers two elliptic curves

$$
y^{2}=x^{3}-x \quad \text { and } \quad y^{2}=x^{3}-x
$$

one deduces that each of the two numbers

$$
\int_{0}^{1} \frac{d x}{\sqrt{x-x^{3}}}=\frac{1}{2} \mathrm{~B}(1 / 4,1 / 2)=\frac{\Gamma(1 / 4)^{2}}{2^{3 / 2} \pi^{1 / 2}}
$$

and

$$
\int_{0}^{1} \frac{d x}{\sqrt{1-x^{3}}}=\frac{1}{3} \mathrm{~B}(1 / 3,1 / 2)=\frac{\Gamma(1 / 3)^{3}}{2^{4 / 3} 3^{1 / 2} \pi}
$$

is transcendental.

These two formulae (compare with [41] p.21) are special cases of the ChowlaSelberg formula (cf [34] and [38] § 2.3) which expresses the periods of CM elliptic curves as products of values of the Euler Gamma function

$$
\Gamma(z)=e^{-\gamma z} z^{-1} \prod_{n=1}^{\infty}\left(1+\frac{z}{n}\right)^{-1} e^{z / n} .
$$

The extension of Chowla-Selberg formula by G. Shimura to Abelian varieties of CM type gives rise to the relations of Deligne-Koblitz-Ogus on Gamma function (see [14]). 
2.3. Genus $\geq 1$ : Abelian integrals. In [52], Th. Schneider proves similar results for Abelian integrals. His proof is an extension in several variables of his previous arguments for the one dimensional case. In the analytic part of the proof, the main tool is Schwarz Lemma. He proves a several variables version of Schwarz' Lemma by means of an interpolation formula for Cartesian products. This method enables him to prove results on Abelian functions. The most important example of results he obtains is the following:

Theorem 12. Let $a$ and $b$ be rational numbers which are not integers and such that $a+b$ is not an integer. Then the number

$$
\mathrm{B}(a, b)=\frac{\Gamma(a) \Gamma(b)}{\Gamma(a+b)}=\int_{0}^{1} x^{a-1}(1-x)^{b-1} d x
$$

is transcendental.

Many papers deal with the arithmetic nature of Abelian integrals: the works by Th. Schneider in 1940 have been pursued first by S. Lang in the 1960's, then by D.W. Masser using Baker's method in the 1980's, until an essentially complete solution of the question was reached in 1989 by G. Wüstholz [65]. He proved a generalization of Baker's Theorem 4 to commutative algebraic groups. Essentially we know the answer to the questions of transcendence and linear independence over the field of algebraic numbers of periods of Abelian integrals of the first, the second or the third kind. For instance J. Wolfart and G. Wüstholz [64] have shown that the only linear dependence relations with algebraic coefficients between the values $\mathrm{B}(a, b)$ of Euler Beta function at points $(a, b) \in \mathbf{Q}^{2}$ are those which follow from the Deligne-Koblitz-Ogus relations.

Moreover we know quantitative estimates which give lower bounds for an Abelian integral which is not zero. The most recent and sharp estimates in this context for the general situation of commutative algebraic groups are due to É. Gaudron [28, 29].

Linear relations with algebraic coefficients between Abelian integrals are well understood now; , we cannot say the same so far for algebraic relations. In a footnote [35], A. Grothendieck suggests a conjectural statement on the transcendence of periods of Abelian varieties over the field of algebraic numbers. The first precise formulation of this conjecture in the projective smooth case is given by S. Lang in his book [39] on transcendental numbers (see also [22]); in the same book is given for the first time the statement of Schanuel's Conjecture on the algebraic independence of values of the exponential function (which includes the conjecture 43 on algebraic independence of logarithms of algebraic numbers). This topic has been revisited by Y. André (see $[6,7]$ ) who proposes his generalized periods Conjecture ([7] 24.3.1) which contains both the Conjectures of Grothendieck and Schanuel. The recent booklet [7] contains a detailed exposition of Grothendieck's Conjecture, and its various versions (including the one in [38]) and extensions. 
For 1-motives attached to products of elliptic curves, C. Bertolin [9] rephrased André's conjecture more explicitly by stating her elliptico-toric conjecture, which contains the expected transcendence and algebraic independence results concerning the values of the exponential function, of the Weierstrass $\wp$ and $\zeta$ functions, of elliptic integrals and of the modular invariant $j$ (see also $[60,61]$ ).

\section{Values of the Euler Gamma function}

From the definition (13) of Euler Beta function as an integral, it follows that its values at points in $\mathbf{Q}^{2}$ where it is defined are periods. From the relation (13) between Gamma and Beta functions, one deduces

$$
\Gamma\left(a_{1}\right) \cdots \Gamma\left(a_{n}\right)=\Gamma\left(a+\cdots+a_{n}\right) \prod_{i=1}^{n-1} \mathrm{~B}\left(a_{1}+\cdots+a_{i-1}, a_{i}\right) .
$$

Hence for any $p / q \in \mathbf{Q}$ with $p>0$ and $q>0$, the number $\Gamma(p / q)^{q}$ is a period. For instance

$$
\pi=\Gamma(1 / 2)^{2}=\int_{0}^{1} x^{-1 / 2}(1-x)^{-1 / 2} d x .
$$

From (9) and (10) one derives also expressions for $\Gamma(1 / 3)^{3}$ and $\Gamma(1 / 4)^{4}$ as periods.

The arithmetic nature of the values of Euler Beta function at rational points a much better understood (thanks to Schneider's Theorem 12) than the values of the Gamma function. The fact that the number $\Gamma(1 / 2)=\sqrt{\pi}$ is transcendental follows from Lindemann's Theorem on the transcendence of $\pi$. The transcendence of the numbers $\Gamma(1 / 4)$ and $\Gamma(1 / 3)$ has been proved in 1976 by G.V. Cudnovs'kiu [20].

Theorem 14. The two numbers

$$
\Gamma(1 / 4) \text { and } \pi
$$

are algebraically independent, and the same is true for the two numbers

$$
\Gamma(1 / 3) \text { and } \pi \text {. }
$$

As noticed by D.W. Masser, one can formulate this statement by saying that the two numbers $\Gamma(1 / 4)$ and $\Gamma(1 / 2)$ are algebraically independent, and the same for $\Gamma(1 / 3)$ and $\Gamma(2 / 3)$.

From the standard relations (see (20) below) satisfied by the Gamma function one deduces other transcendence results; for instance $\Gamma(1 / 6)$ is also a transcendental numbers. Apart from these values, no proof of the transcendence of another value of the Gamma function at a rational point is known. Transcendence results are bound to denominators which divide 6: for other values the genus of the corresponding Fermat curve is larger than 1, and so far the transcendence results on Abelian varieties are not as strong as their elliptic counterparts. 
The proof by G.V. Cudnovs'kii of his Theorem 14 rests on the following result [20], dealing with periods and quasi-periods of Weierstrass functions, which he applies to the elliptic curves $y^{2}=x^{3}-x$ and $y^{2}=x^{3}-1$ using (9) and (10)

Let $\wp$ be a Weierstrass elliptic function with invariants $g_{2}$ and $g_{3}$. Let $\omega$ be a nonzero period of $\wp$ and let $\eta$ be the corresponding quasi-period of the $\zeta$ function:

$$
\wp^{\prime 2}=4 \wp^{3}-g_{2} \wp-g_{3}, \quad \zeta^{\prime}=-\wp, \quad \zeta(z+\omega)=\zeta(z)+\eta .
$$

Then two at least of the numbers

$$
g_{2}, g_{3}, \omega / \pi, \eta / \pi
$$

are algebraically independent.

From this statement one deduces also that the number (8) is transcendental, not only over $\mathbf{Q}$, but also over the field $\mathbf{Q}(\pi)$ (cf. [38] $\S 1.1$ ).

So far the only known proof of the transcendence (over $\mathbf{Q}$ ) of $\Gamma(1 / 4)$ as well as of $\Gamma(1 / 3)$ is Čudnovs'kiı̌'s one which establishes the stronger result that these numbers are transcendental over $\mathbf{Q}(\pi)$.

From the quantitative point of view, sharp transcendence measures for these numbers have been obtained by P. Philippon and then by S. Bruiltet [15]:

Theorem 15. Let $P \in \mathbf{Z}[X, Y]$ be a non-constant polynomial of degree $d$ and height $H$. Then

$$
\log \mid P\left(\pi, \Gamma(1 / 4) \mid>-10^{326}\left((\log H+d \log (d+1)) d^{2}(\log (d+1))^{2}\right.\right.
$$

and

$$
\log \mid P\left(\pi, \Gamma(1 / 3) \mid>-10^{330}\left((\log H+d \log (d+1)) d^{2}(\log (d+1))^{2} .\right.\right.
$$

This shows that neither $\Gamma(1 / 4)$ nor $\Gamma(1 / 3)$ is a Liouville number.

One might expect that the next step could be the transcendence of the number $\Gamma(1 / 5)$ (cf. [41], p. 2 and p. 35). From Schneider's Theorem 12 one deduces the transcendence of the number $\mathrm{B}(1 / 5,1 / 5)$, hence one at least of the two numbers $\Gamma(1 / 5), \Gamma(2 / 5)$ is transcendental. A stronger statement follows from the work of P. Grinspan [33] (see also [58]):

Theorem 16. One at least of the two numbers $\Gamma(1 / 5), \Gamma(2 / 5)$ is transcendental over the field $\mathbf{Q}(\pi)$.

In other terms, two at least of the three numbers $\Gamma(1 / 5), \Gamma(2 / 5)$ and $\pi$ are algebraically independent. The proof of [33] also yields a quantitative result.

Since the Fermat curve $x^{5}+y^{5}=z^{5}$ with exponent 5 has genus 2, its Jacobian is an Abelian surface; to prove Theorem 16, one needs to replace, in Cudnovs'kilı's proof, the elliptic functions by Abelian functions; this explains the difficulty to 
separate the two numbers $\Gamma(1 / 5)$ and $\Gamma(2 / 5)$ when one is interested to prove the transcendence of one of them.

Before continuing with the denominator 5, let us come back briefly to the denominators 3 and 4 . Theorem 14 has been extended by Yu.V. Nesterenko $[42,43]$ in 1996 , who succeeded to obtain the algebraic independence of three numbers:

Theorem 17. The three numbers

$$
\Gamma(1 / 4), \quad \pi \quad \text { and } \quad e^{\pi}
$$

are algebraically independent, and the same is true with the three numbers

$$
\Gamma(1 / 3), \quad \pi \text { and } e^{\pi \sqrt{3}} .
$$

To prevent confusion, let us mention that the numbers $e^{\pi}$ and $e^{\pi \sqrt{3}}$ are not expected to be periods.

The proof by Yu.V. Nesterenko of his Theorem 17 involves Eisenstein series $E_{2}$, $E_{4}$ and $E_{6}$ - here we shall use the notations $P, Q, R$ introduced by S. Ramanujan:

$$
\left\{\begin{array}{l}
P(q)=E_{2}(q)=1-24 \sum_{n=1}^{\infty} \frac{n q^{n}}{1-q^{n}} \\
Q(q)=E_{4}(q)=1+240 \sum_{n=1}^{\infty} \frac{n^{3} q^{n}}{1-q^{n}} \\
R(q)=E_{6}(q)=1-504 \sum_{n=1}^{\infty} \frac{n^{5} q^{n}}{1-q^{n}}
\end{array}\right.
$$

The first transcendence results on the values of these functions are due to D. Bertrand in the 1970's. A remarkable progress was achieved in 1996 by K. Barré-Sirieix, G. Diaz, F. Gramain and G. Philibert [8], who solved the following problem raised by K. Mahler (and by Yu.V. Manin in the $p$-adic case) on the modular function $J=Q^{3} / \Delta$, where $\Delta=12^{-3}\left(Q^{3}-R^{2}\right)$ : for any $q \in \mathbf{C}$ with $0<|q|<1$, one at least of the two numbers $q, J(q)$ is transcendental.

This breakthrough enabled Yu.V. Nesterenko [42] to prove the next result, which is quoted in $\S 2.4$ of [38]:

Let $q \in \mathbf{C}$ be a complex number satisfying $0<|q|<1$. Then three at least of the four numbers

$$
q, P(q), Q(q), R(q)
$$

are algebraically independent. 
Theorem 17 follows by specializing $q=e^{-2 \pi}$ and $q=-e^{-\pi \sqrt{3}}$, since $J\left(e^{-2 \pi}\right)=$ 1728 ,

$$
P\left(e^{-2 \pi}\right)=\frac{3}{\pi}, \quad Q\left(e^{-2 \pi}\right)=3\left(\frac{\omega}{\pi}\right)^{4}, \quad R\left(e^{-2 \pi}\right)=0
$$

with (cf. (9))

$$
\omega=\frac{\Gamma(1 / 4)^{2}}{\sqrt{8 \pi}}=2.6220575542 \ldots
$$

while $J\left(-e^{-\pi \sqrt{3}}\right)=0$,

$$
P\left(-e^{-\pi \sqrt{3}}\right)=\frac{2 \sqrt{3}}{\pi}, \quad Q\left(-e^{-\pi \sqrt{3}}\right)=0, \quad R\left(-e^{-\pi \sqrt{3}}\right)=\frac{27}{2}\left(\frac{\omega^{\prime}}{\pi}\right)^{6}
$$

with (cf. (10))

$$
\omega^{\prime}=\frac{\Gamma(1 / 3)^{3}}{2^{4 / 3} \pi}=2.428650648 \ldots
$$

In [38] $\S 2.3$ there are comments on relations between periods and Eisenstein series (as well as theta functions, which also occur in the work [42] of Nesterenko — see [43]).

Nesterenko's Theorem 17 and Grinspan's Theorem 16 suggest the following statements, which is likely to be a special case of André's extension of Grothen -dieck's Conjecture (see $\S 2$ and [7] 23.4.3):

Conjecture 19. Three at least of the four numbers

$$
\Gamma(1 / 5), \quad \Gamma(2 / 5), \quad \pi \quad \text { and } \quad e^{\pi \sqrt{5}}
$$

are algebraically independent.

This problem has been considered by F. Pellarin (see [47]).

A more ambitious goal is to give a complete list of all algebraic relations among the values of the Gamma function at rational points. D. Rohrlich considered the question for multiplicative relations. Let us first recall what are the standard relations. For any $a \in \mathbf{C}$ which is not a pole of $\Gamma(x), \Gamma(x+1), \Gamma(1-x)$ nor of $\Gamma(n x)$

$$
\begin{array}{cc}
\text { (Translation) } & \Gamma(a+1)=a \Gamma(a), \\
\text { (Reflection) } & \Gamma(a) \Gamma(1-a)=\frac{\pi}{\sin (\pi a)} \\
\text { (Multiplication) } & \left\{\begin{array}{l}
\prod_{k=0}^{n-1} \Gamma\left(a+\frac{k}{n}\right)=(2 \pi)^{(n-1) / 2} n^{-n a+(1 / 2)} \Gamma(n a) \\
\text { for any positive integer } n .
\end{array}\right.
\end{array}
$$


Conjecture 21. (D. Rohrlich). Any multiplicative dependence relation of the form

$$
\pi^{b / 2} \prod_{a \in \mathbf{Q}} \Gamma(a)^{m_{a}} \in \overline{\mathbf{Q}}
$$

with $b$ and $m_{a}$ in $\mathbf{Z}$ is a consequence of the standard relations (20).

A formalization of this Conjecture in terms of "universal distribution" has been proposed by S. Lang in [40].

A stronger conjecture than 21 is the Rohrlich-Lang Conjecture which deals not only with monomial relations, but more generally with polynomial relations.

Conjecture 22. (Rohrlich-Lang). The ideal over $\overline{\mathbf{Q}}$ of all algebraic relations among values of the function $(1 / \sqrt{2 \pi}) \Gamma(a)$ for $a \in \mathbf{Q}$ is generated by the distribution relations, the functional equation and the oddness of the Gamma function.

The precise relation between conjectures 21, 22 and the Grothendieck's Conjecture (for certain Abelian varieties) is explained in detail in [7] 24.6.

\section{Values of the Riemann zeta function}

We start with an elementary result on the values of the Riemann zeta function at the positive integers.

Lemma 23. For $s \geq 2$, the number

$$
\zeta(s)=\sum_{n \geq 1} \frac{1}{n^{s}}
$$

is a period.

Proof: The formula

$$
\zeta(s)=\int_{1>t_{1}>\cdots>t_{s}>0} \frac{d t_{1}}{t_{1}} \cdots \frac{d t_{s-1}}{t_{s-1}} \cdot \frac{d t_{s}}{1-t_{s}}
$$

is easily checked.

It will be convenient to use the notation of iterated Chen integrals ([17] § 2.6) and to write (24) as

$$
\zeta(s)=\int_{0}^{1} \omega_{0}^{s-1} \omega_{1} \quad \text { with } \quad \omega_{0}=\frac{d t}{t} \quad \text { and } \quad \omega_{1}=\frac{d t}{1-t} .
$$

The arithmetic nature of the values of the Riemann zeta function at positive even integers is known since Euler:

$$
\pi^{-2 k} \zeta(2 k) \in \mathbf{Q} \quad \text { for } \quad k \geq 1 .
$$


These rational numbers involve Bernoulli numbers (see for instance formula (38) in $[17])$.

Lindemann's Theorem asserts that the number $\pi$ is transcendental, hence so is $\zeta(2 k)$ for any integer $k \geq 1$.

To prove that given numbers are rational or algebraic is most often more fruitful than to prove that they are transcendental. For instance, in the case of Euler's formula (26), the Bernoulli numbers carry a lot of interesting arithmetic information. However, our purpose here is to give the state of the art on transcendence results: the goal of transcendence statements is to ensure that all the algebraic relations are known, hence that the source of all arithmetic information is already available.

The main Diophantine question raised by Euler numbers is to decide which are the algebraic relations among the numbers

$$
\zeta(2), \quad \zeta(3), \quad \zeta(5), \quad \zeta(7) \ldots ?
$$

One expects that there are no relation at all ([17] and [27] Conjecture 0.1). In other terms:

Conjecture 27. The numbers

$$
\zeta(2), \quad \zeta(3), \quad \zeta(5), \quad \zeta(7) \ldots
$$

are algebraically independent.

Very few is known on this conjecture. In $1978 \mathrm{R}$. Apéry proved that the number $\zeta(3)$ is irrational . Apéry's proof shows that the number $\zeta(3)$ is not a Liouville number. The sharpest known irrationality measure for $\zeta(3)$ is due to G. Rhin and C. Viola [49]:

$$
\left|\zeta(3)-\frac{p}{q}\right|>q^{-\mu}
$$

for sufficiently large $q$, with $\mu=5,513 \ldots$

The recent works initiated by T. Rivoal and followed by T. Rivoal, K. Ball and W. Zudilin among others, provide the first information on the arithmetic nature of the values of the Riemann zeta function at odd positive integers. In particular the dimension of the $\mathbf{Q}$-vector space spanned by the number $\zeta(2 k+1), k \geq 1$ is infinite (cf. [27]).

A preliminary step towards a proof of Conjecture 27 is to linearize the situation. Indeed, available Diophantine methods for proving linear independence are much more efficient than methods for proving algebraic independence. An example of this claim is Baker's Theorem 4 which solves the questions of linear independence over the field of algebraic numbers of logarithms of algebraic numbers, while it is not known that there exists two algebraically independent logarithms of algebraic 
numbers. An earlier example, showing the power of linear independence methods, is the Lindemann-Weierstraß' Theorem, which can be stated either as a result of algebraic independence, ([26] Th. 2.3'), or as a result of linear independence ([26] Th. 2.3) - the proof by F. Lindemann et K. Weierstraß is a proof of linear independence.

Euler already noticed that the product of two values of the Riemann zeta function (as it is called now) is again the sum of a series. Indeed, from the relation

$$
\sum_{n_{1} \geq 1} n_{1}^{-s_{1}} \sum_{n_{2} \geq 1} n_{2}{ }^{-s_{2}}=\sum_{n_{1}>n_{2} \geq 1} n_{1}^{-s_{1}} n_{2}^{-s_{2}}+\sum_{n_{2}>n_{1} \geq 1} n_{2}^{-s_{2}} n_{1}^{-s_{1}}+\sum_{n \geq 1} n^{-s_{1}-s_{2}},
$$

one deduces, for $s_{1} \geq 2$ and $s_{2} \geq 2$,

$$
\zeta\left(s_{1}\right) \zeta\left(s_{2}\right)=\zeta\left(s_{1}, s_{2}\right)+\zeta\left(s_{2}, s_{1}\right)+\zeta\left(s_{1}+s_{2}\right)
$$

with

$$
\zeta\left(s_{1}, s_{2}\right)=\sum_{n_{1}>n_{2} \geq 1} n_{1}^{-s_{1}} n_{2}^{-s_{2}} .
$$

For $k, s_{1}, \ldots, s_{k}$ positive integers with $s_{1} \geq 2$, we set $\underline{s}=\left(s_{1}, \ldots, s_{k}\right)$ and

$$
\zeta(\underline{s})=\sum_{n_{1}>n_{2}>\cdots>n_{k} \geq 1} \frac{1}{n_{1}^{s_{1}} \cdots n_{k}^{s_{k}}} .
$$

These numbers are called "Multiple Zeta Values" or "MZV". For $k=1$ we recover of course Euler's numbers $\zeta(s)$.

Remark 29. Each of the numbers $\zeta(\underline{s})$ is a period: indeed, using the iterated Chen integrals notation (25) we have (cf. [17] § 2.6):

$$
\zeta(\underline{s})=\int_{0}^{1} \omega_{0}^{s_{1}-1} \omega_{1} \cdots \omega_{0}^{s_{k}-1} \omega_{1} .
$$

The product of series (28) is a linear combination, with integer coefficients, of such series. Therefore the vector space (over $\mathbf{Q}$ or over $\overline{\mathbf{Q}}$ ) spanned by the numbers $\zeta(\underline{s})$ is also an algebra over the same field. Moreover, the product of two integrals (30) is also a linear combination of such integrals. These linear combinations are not the same as the relations occurring from the product of two series: therefore, taking the difference yields nontrivial linear relations with integer coefficients between MZV. Further linear relations can be derived - for instance Euler's relation $\zeta(2,1)=\zeta(3)$ - by taking into account the relations which are obtained using the same argument for divergent series and using a regularization argument [17].

A complete description of all linear relations among MZV should in principle yield a description of all algebraic relations among these numbers, hence should provide a solution to Conjecture 27 concerning the algebraic independence, over 
the field $\mathbf{Q}(\pi)$, of the values at odd positive integers of the Riemann zeta function. The contrast is interesting between the situation suggested on the one hand by Conjecture 27, where no algebraic relation is expected, and where the underlying algebraic structure is uninteresting, and on the other hand by the rich structure provided by the known linear relations between MZV.

The goal now is to describe all linear relations among MZV. Let $\mathfrak{Z}_{p}$ be the Q-subspace of $\mathbf{R}$ spanned by the numbers $\zeta(\underline{s})$ for $\underline{s}$ of "weight" $s_{1}+\cdots+s_{k}=p$, with $\mathfrak{Z}_{0}=\mathbf{Q}$ and $\mathfrak{Z}_{1}=\{0\}$.

Here is Zagier's Conjecture (Conjecture 108 of [17]) on the dimension $d_{p}$ of $\mathfrak{Z}_{p}$. The precise relation between conjectures 21, 22 and the Grothendieck's Conjecture (for certain mixed Tate motives) is explained in detail in [7] 25.4 and 25.7. See also the paper by P. Deligne and A.B. Goncharov [23].

Conjecture 31. The numbers $d_{p}$ satisfy, for $p \geq 3$,

$$
d_{p}=d_{p-2}+d_{p-3} \text {. }
$$

Hence the sequence $\left(d_{0}, d_{1}, d_{2}, \ldots\right)$ starts with $(1,0,1,1,1,2,2, \ldots)$.

This conjecture can be written

$$
\sum_{p \geq 0} d_{p} X^{p}=\frac{1}{1-X^{2}-X^{3}} .
$$

A candidate for a basis of the space $\mathfrak{Z}_{p}$ is suggested by M. Hoffman ([36], Conjecture $\mathrm{C})$ :

Conjecture 32. $A$ basis of $\mathfrak{Z}_{p}$ over $\mathbf{Q}$ is given by the numbers $\zeta\left(s_{1}, \ldots, s_{k}\right)$, where $s_{1}+\cdots+s_{k}=p$ and each $s_{i}$ is either 2 or 3 .

This conjecture is compatible with what is known for $p \leq 16$ (work of Hoang Ngoc Minh and others). For instance, if $\{a\}_{b}$ denotes the sequence of $b$ occurences of $a$, it is expected that the 7 following values

$$
\begin{gathered}
\zeta\left(\{2\}_{5}\right), \zeta\left(\{2\}_{2},\{3\}_{2}\right), \zeta\left(\{2,3\}_{2}\right), \zeta\left(\left(2,\{3\}_{2}, 2\right),\right. \\
\zeta\left(3,\{2\}_{2}, 3\right), \zeta\left(\{3,2\}_{2}\right), \zeta\left(\{3\}_{2},\{2\}_{2}\right)
\end{gathered}
$$

provide a basis of the vector space $\mathfrak{Z}_{10}$.

Exemple 33. Here are the first values of $d_{p}$ :

- $d_{0}=1$ because we agree that $\zeta\left(s_{1}, \ldots, s_{k}\right)=1$ for $k=0$.

- $d_{1}=0$ since $\left\{\left(s_{1}, \ldots, s_{k}\right) ; s_{1}+\cdots+s_{k}=1, s_{1} \geq 2\right\}=\emptyset$.

- $d_{2}=1$ since $\zeta(2) \neq 0$

- $d_{3}=1$ since $\zeta(2,1)=\zeta(3) \neq 0$ 
- $d_{4}=1$ since $\zeta(4) \neq 0$ and

$$
\zeta(3,1)=\frac{1}{4} \zeta(4), \quad \zeta(2,2)=\frac{3}{4} \zeta(4), \quad \zeta(2,1,1)=\zeta(4)=\frac{2}{5} \zeta(2)^{2} .
$$

The first value of $d_{p}$ which is not known is $d_{5}$. Conjecture 31 predicts $d_{5}=2$, and we know $d_{5} \in\{1,2\}$ because

$$
\begin{aligned}
\zeta(2,1,1,1) & =\zeta(5), \\
\zeta(3,1,1) & =\zeta(4,1)=2 \zeta(5)-\zeta(2) \zeta(3), \\
\zeta(2,1,2) & =\zeta(2,3)=\frac{9}{2} \zeta(5)-2 \zeta(2) \zeta(3), \\
\zeta(2,2,1) & =\zeta(3,2)=3 \zeta(2) \zeta(3)-\frac{11}{2} \zeta(5)
\end{aligned}
$$

Hence $d_{5}=2$ is equivalent to the irrationality of the number $\zeta(2) \zeta(3) / \zeta(5)$.

Conjecture 31 predicts an exact value for the dimension $d_{p}$ of $\mathfrak{Z}_{p}$. The Diophantine open problem is to prove the lower bound. The upper bound has been established recently thanks to the work of A.B. Goncharov [30] and T. Terasoma [57] (see also Theorem 6.4 of [36] and Cor. 5.25 of [23]. ):

The integers $\delta_{p}$ which are defined by the linear recurrence relation of Zagier's Conjecture 31

$$
\delta_{p}=\delta_{p-2}+\delta_{p-3}
$$

with initial conditions $\delta_{0}=1, \delta_{1}=0$ give an upper bound for the dimension $d_{p}$ of $\mathfrak{Z}_{p}$.

Surprisingly enough, this bound is not obtained through a combinatorial study of the known relations between MZV, but by a fairly general motivic argument.

\section{Hypergeometric FUnCTIONS}

For $a, b, c$ and $z$ complex numbers with $c \notin \mathbf{Z}_{\leq 0}$ and $|z|<1$, Gauss hypergeometric function (see for instance [26], Chap. $1 \S 3.6$, Chap. $2 \S 3.2$ ) is defined by

where

$$
{ }_{2} F_{1}(a, b ; c \mid z)=\sum_{n=0}^{\infty} \frac{(a)_{n}(b)_{n}}{(c)_{n}} \cdot \frac{z^{n}}{n !}
$$

$$
(a)_{n}=a(a+1) \cdots(a+n-1) .
$$

Exemple 34. Let $K, P_{n}$ and $T_{n}$ denote respectively Jacobi's elliptic integral of the first kind, the $n$-th Legendre polynomial and the $n$-th Chebyshev polynomial:

$$
K(z)=\int_{0}^{1} \frac{d x}{\sqrt{\left(1-x^{2}\right)\left(1-z^{2} x^{2}\right)}}, \quad P_{n}(z)=\frac{1}{n !}\left(\frac{d}{d z}\right)^{n}\left(1-z^{2}\right)^{n}
$$




$$
T_{n}(\cos z)=\cos (n z)
$$

Then:

$$
\left\{\begin{array}{cl}
{ }_{2} F_{1}(a, 1 ; 1 \mid z) & =\frac{1}{(1-z)^{a}}, \\
{ }_{2} F_{1}(1,1 ; 2 \mid z) & =\frac{1}{z} \log (1+z), \\
{ }_{2} F_{1}\left(1 / 2,1 ; 3 / 2 \mid z^{2}\right) & =\frac{1}{2 z} \log \frac{1+z}{1-z}, \\
{ }_{2} F_{1}\left(1 / 2,1 / 2 ; 3 / 2 \mid z^{2}\right) & =\frac{1}{z} \arcsin z, \\
{ }_{2} F_{1}\left(1 / 2,1 / 2 ; 1 \mid z^{2}\right) & =\frac{2}{\pi} K(z), \\
{ }_{2} F_{1}(-n, n+1 ; 1 \mid(1+z) / 2) & =2^{-n} P_{n}(z), \\
{ }_{2} F_{1}(-n, n ; 1 / 2 \mid(1+z) / 2) & =(-1)^{n} T_{n}(z) .
\end{array}\right.
$$

For $c>b>0$ rational numbers, we have (Euler, 1748)

$$
{ }_{2} F_{1}(a, b ; c \mid z)=\frac{\Gamma(c)}{\Gamma(b) \Gamma(c-b)} \int_{0}^{1} t^{b-1}(1-t)^{c-b-1}(1-t z)^{-a} d t
$$

using Gamma's reflexion formula in (20) together with the connection (13) between the Beta and Gamma function, one deduces

$$
\frac{\Gamma(c)}{\Gamma(b) \Gamma(c-b)} \in \frac{1}{\pi} \mathcal{P} .
$$

Therefore [38] § 2.2, for $a, b, c$ rational numbers satisfying $c \notin \mathbf{Z}_{\leq 0}$ and for $z \in \overline{\mathbf{Q}}$ with $|z|<1$, we have

$$
{ }_{2} F_{1}(a, b ; c \mid z) \in \frac{1}{\pi} \mathcal{P} .
$$

Recall $\mathcal{P} \subset(1 / \pi) \mathcal{P}$. A suggestion of [38] $\S 2.2$ is that under the same conditions, ${ }_{2} F_{1}(a, b ; c \mid z)$ does not belong to $\mathcal{P}$.

Remark 37. According to Gauss, for $a, b$, $c$ real numbers with $c>a+b$ and $c \notin \mathbf{Z}_{\leq 0}$, we have

$$
{ }_{2} F_{1}(a, b ; c \mid 1)=\frac{\Gamma(c-a) \Gamma(c-b)}{\Gamma(c) \Gamma(c-a-b)} .
$$

Remark 38. A remarkable relation connecting the modular invariant $j(z)=$ $J\left(e^{2 i \pi z}\right)$ and Eisenstein series $E_{4}=Q(c f(18))$ is the following [38] $\S 2.3$, due to Fricke and Klein:

$$
{ }_{2} F_{1}\left(\frac{1}{12}, \frac{5}{12} ; 1 \mid \frac{1728}{j(z)}\right)=Q(z)^{1 / 4}
$$


The transcendence of the values ${ }_{2} F_{1}(a, b ; c \mid z)$ of hypergeometric functions when $a, b, c$ and $z$ are rational numbers has been investigated as early as 1929 by C.L. Siegel [55]. A.B. Shidlovskii and his school produced a number of important results on this matter (see [26]).

In 1988, J. Wolfart [62] studied the set $\mathcal{E}$ of algebraic numbers $\xi$ such that ${ }_{2} F_{1}(\xi)$ is also an algebraic number. When ${ }_{2} F_{1}$ is an algebraic function, $\mathcal{E}=\overline{\mathbf{Q}}$ is the set of all algebraic numbers. Assume now that ${ }_{2} F_{1}$ is a transcendental function. J. Wolfart [62] proved that there is a bijective map between the set $\mathcal{E}$ and a set of Abelian varieties of CM type - this is an extension to higher dimension of a theorem due to Th. Schneider on the transcendence of the modular invariant $j$ ([51], [53] Th. 17).

Wolfart 's proof uses the fact that the numbers ${ }_{2} F_{1}(a, b, c ; z)$ are related with the periods of differential forms on the curve

$$
y^{N}=x^{A}(1-x)^{B}(1-z x)^{C}
$$

with $A=(1-b) N, B=(b+1-c) N, C=a N$, while $N$ is the smallest common denominator of $a, b, c$ (see [37]). His transcendence tool is the analytic subgroup Theorem of Wüstholz [65].

J. Wolfart was able to give a complete list of algebraic numbers among these hypergeometric values. In the course of his study, he found with F. Beukers [10] new relations which apparently were not stated before, like

$$
{ }_{2} F_{1}\left(\frac{1}{12}, \frac{5}{12} ; \frac{1}{2} \mid \frac{1323}{1331}\right)=\frac{3}{4} \sqrt[4]{11}
$$

and

$$
{ }_{2} F_{1}\left(\frac{1}{12}, \frac{7}{12} ; \frac{2}{3} \mid \frac{64000}{64009}\right)=\frac{2}{3} \sqrt[6]{253}
$$

When the monodromy group of the hypergeometric differential equation satisfied by ${ }_{2} F_{1}$ is an arithmetic triangular group, the set $\mathcal{E}$ is infinite. Wolfart [62] claimed that conversely, the set $\mathcal{E}$ is finite when the monodromy group is not arithmetic but his proof was not complete. The works of P. Cohen and J. Wolfart [18], then of P. Cohen and G. Wüstholz [19], provide a link between this question and the André-Oort Conjecture [5, 45], according to which the special subvarieties of the Shimura varieties are precisely the subvarieties which contain a Zariski dense subset of special points. P. Cohen then noticed that a special case of the André-Oort Conjecture in dimension 1 suffices; the crucial fact has been proved by B. Edixhoven and A. Yafaev [25]: in a Shimura variety, a curve contains infinitely many points on the same Hecke orbit of a special point if and only if it is special. This provides an answer to Siegel's initial question. More precisely, if we combine the results of $[18,19,25,62]$, one deduces: there is a bijective map between the exceptional set $\mathcal{E}$ and a set of points in the same Hecke 
orbit of a special (CM) point on a curve in a Shimura variety which is defined over $\overline{\mathbf{Q}}$. The set $\mathcal{E}$ is infinite when the monodromy group is arithmetic, it is finite when the monodromy group is not arithmetic.

To Gauss hypergeometric function is associated Gauss continued fraction

$$
\begin{aligned}
G(z) & =G(a, b, c ; z)={ }_{2} F_{1}(a, b+1 ; c+1 \mid z) /{ }_{2} F_{1}(a, b ; c \mid z) \\
& =1 /\left(1-g_{1} z /\left(1-g_{2} z /(\cdots)\right)\right)
\end{aligned}
$$

with coefficients

$$
\begin{aligned}
g_{2 n-1} & =(a+n-1)(c-b+n-1) /((c+2 n-2)(c+2 n-1)), \\
g_{2 n} & =(b+n)(c-a+n) /((c+2 n-1)(c+2 n)) .
\end{aligned}
$$

J. Wolfart [63] showed that if the parameters $a, b, c$ are rational numbers, $c \neq 0$, $-1,-2, \cdots$, and if $G(z)$ is not an algebraic function, then, for almost all algebraic values of the argument $z$, the value $G(z)$ is a transcendental number. He uses Wüstholz's Theorem [65] together with the results of G. Shimura and Y. Taniyama on Abelian varieties.

For the number we just considered, which are related to hypergeometric functions, when a transcendence proof is known, an effective measure of Diophantine approximation by algebraic numbers is also available (see in particular [26] and $[28,29])$.

Gauss hypergeometric functions are particular members of a much more general family, which is constituted by the generalized hypergeometric functions (see for instance [26] Chap. 2, §6).

Let $p \geq 2$ be an integer, $a_{1}, \ldots, a_{p}, b_{1}, \ldots, b_{p-1}$ and $z$ complex numbers with $b_{i} \notin \mathbf{Z}_{\leq 0}$ and $|z|<1$. Set

$$
{ }_{p} F_{p-1}\left(\begin{array}{c}
a_{1}, \ldots, a_{p} \\
b_{1}, \ldots, b_{p-1}
\end{array} \mid z\right)=\sum_{n=0}^{\infty} \frac{\left(a_{1}\right)_{n} \cdots\left(a_{p}\right)_{n}}{\left(b_{1}\right)_{n} \cdots\left(b_{p-1}\right)_{n}} \cdot \frac{z^{n}}{n !} .
$$

Exemple 39. The functions

$$
{ }_{1} F_{0}\left({ }^{1 / n} \mid z^{n}\right)=\sqrt[n]{1-z^{n}}
$$

and

$$
{ }_{3} F_{2}\left(\begin{array}{c|c}
1 / 4,1 / 2,3 / 4 & 2^{8} z \\
1 / 3,2 / 3 & \frac{3^{3}}{1 / 3}
\end{array}\right)=\sum_{k=0}^{\infty}\left(\begin{array}{c}
4 k \\
k
\end{array}\right) z^{k}
$$

are algebraic.

In connection with these examples, let us quote the main result of a recent Oberwolfach report on integral ratios of factorials and algebraic hypergeometric 
functions by F. Rodriguez-Villegas. Let $\gamma=\left(\gamma_{\nu}\right)_{\nu \geq 1}$ be a sequence of integers which are zero except for finitely many. Define

$$
u_{n}=\prod_{\nu \geq 1}(\nu n) !^{\gamma_{\nu}} .
$$

Assume $\gamma \neq 0$ and

$$
\sum_{\nu \geq 1} \nu \gamma_{\nu}=0
$$

which, by Stirling's formula, is equivalent to the generating series

$$
u:=\sum_{\nu \geq 1} u_{n} \lambda^{n}
$$

having finite non-zero radius of convergence. Set

$$
d:=-\sum_{\nu \geq 1} \gamma_{\nu}
$$

F. Rodriguez-Villegas proves that the generating series $u$ is algebraic if and only if $u_{n} \in \mathbf{Z}$ for every $n \geq 0$ and $d=1$.

By induction on $p$ starting from (36) one can check:

Proposition 40. For $a_{1}, \ldots, a_{p}, b_{1}, \ldots, b_{p-1}$ rational numbers with $p \geq 2, b_{i} \notin$ $\mathbf{Z}_{\leq 0}$ and for $z \in \overline{\mathbf{Q}}$ with $|z|<1$, we have

$$
{ }_{p} F_{p-1}\left(\begin{array}{c}
a_{1}, \ldots, a_{p} \\
b_{1}, \ldots, b_{p-1}
\end{array} \mid z\right) \in \frac{1}{\pi^{p-1}} \mathcal{P} .
$$

The reference [26] gives the state of the art on the arithmetic nature of values of generalized hypergeometric functions, both from the qualitative point of view (irrationality, transcendence, linear independence, algebraic independence) and from the quantitative one (approximation measures, linear independence measures, measures of algebraic independence).

\section{MAHLER'S MEASURE OF POLYNOMIALS IN SEVERAL VARIABLES}

Let $P \in \mathbf{C}\left[z_{1}, \ldots, z_{n}, z_{1}^{-1}, \ldots, z_{n}^{-1}\right]$ be a nonzero Laurent polynomial in $n$ variables. Mahler's measure $\mathrm{M}(P)$ and Mahler's logarithmic measure $\mu(P)$ are defined by

$$
\mu(P)=\log \mathrm{M}(P)=\int_{0}^{1} \cdots \int_{0}^{1} \log \left|P\left(e^{2 i \pi t_{1}}, \ldots, e^{2 i \pi t_{n}}\right)\right| d t_{1} \cdots d t_{n} .
$$

In the easiest case $n=1$ we write

$$
P(z)=\sum_{i=0}^{d} a_{d-i} z^{i}=a_{0} \prod_{i=1}^{d}\left(z-\alpha_{i}\right)
$$


and we have

$$
\mathrm{M}(P)=\left|a_{0}\right| \prod_{i=1}^{d} \max \left\{1,\left|\alpha_{i}\right|\right\} .
$$

One deduces $\mu(P) \in \mathcal{P}$.

More generally, for $P \in \overline{\mathbf{Q}}\left[z_{1}, \ldots, z_{n}, z_{1}^{-1}, \ldots, z_{n}^{-1}\right]$ we have

$$
\mu(P) \in \frac{1}{\pi^{n}} \mathcal{P} .
$$

D. Boyd and C.J. Smyth (see the references in [12]) computed several examples of the value of $\mu$ which they expressed in terms of special values of the $L$-function attached to Dirichlet's characters. Then D. Boyd, F. Rodriguez Villegas, V. Maillot and S. Vandervelde wrote some logarithmic Mahler's measures as combinations of the dilogarithm function. Apart from the well known example

$$
\mu\left(1+z_{1}+z_{2}+z_{3}\right)=\frac{7}{2 \pi^{2}} \zeta(3),
$$

due to C.J. Smyth, known results deal mainly with polynomials in two variables. However, thanks to the works of C. Deninger, there is some hope for a better understanding of more general cases.

\section{EXPONENTIAL OF PERIOdS AND EXPONENTIAL PERIODS}

7.1. Exponential of periods. One of the suggestions of Kontsevich and Zagier in [38] $\S 1.2$ is that the numbers $1 / \pi$ and $e$ may not be periods. Among the candidates for being nonperiods one may include $e^{\pi}$ and $e^{\pi^{2}}$.

The transcendence of the number $e^{\pi}$ is known thanks to A.O. Gel'fond in 1929 - it is now also a consequence of Gel'fond-Schneider's solution of Hilbert's seventh problem (hence of Baker's Theorem 4). The transcendence of $e^{\pi^{2}}$ is not yet proved.

Conjecture 41. Let $\alpha_{1}, \alpha_{2}, \alpha_{3}$ be nonzero algebraic numbers. For $j=1,2,3$ let $\log \alpha_{j} \in \mathbf{C} \backslash\{0\}$ be a nonzero logarithms of $\alpha_{j}$, which means a nonzero complex number such that $e^{\log \alpha_{j}}=\alpha_{j}$. Then

$$
\left(\log \alpha_{1}\right)\left(\log \alpha_{2}\right) \neq \log \alpha_{3} \text {. }
$$

Exemple 42. With $\log \alpha_{1}=\log \alpha_{2}=i \pi$, the transcendence of the number $e^{\pi^{2}}$ follows from Conjecture 41. Another example is the transcendence of the number $2^{\log 2}$.

Other conjectures are proposed in [61], both for the exponential function (three, four, five exponentials conjectures) and for elliptic functions. Very few special cases of these conjectures have been proved. The appendix of [61] by H. Shiga provides a link with periods of Kummer surfaces. 
The proofs of the few partial results which are known in the direction of Conjecture 41 and of the four exponentials Conjecture [39, 59, 61] rest on the method which enabled Th. Schneider in 1934 to solve Hilbert's seventh problem. In this method the addition Theorem of the exponential function, namely $e^{x+y}=e^{x} e^{y}$, plays the main role, while the differential equation is not used, in contrast with what is suggested at the end of $\S 2.4$ of [38]. A second example of a transcendence proof which does not use derivatives is by G. Diaz [24] for the special case of Hermite-Lindemann's Theorem on the transcendence of $e^{\beta}$ when $\beta$ is algebraic and $\beta \notin \mathbf{R} \cup i \mathbf{R}$. A third transcendence method where derivatives are not involved is Mahler's one, which is the main topic of K. Nishioka's Lecture Notes [44]. By the way, Conjecture 5.4 of [60] on the transcendence of numbers whose development in a basis is given by an automatic sequence, which had been investigated by J.H. Loxton and A.J. van der Poorten by means of this method of Mahler, has been settled by B. Adamczewski, Y. Bugeaud and F. Lucas $[2,1]$, thanks to completely different arguments based on the very efficient Subspace Theorem of W.M. Schmidt.

Conjecture 41 is a very special case of the Conjecture on algebraic independence of logarithms of algebraic numbers (which is in turn only a special case of Schanuel's Conjecture):

Conjecture 43. (Algebraic independence of logarithms). Logarithms of algebraic numbers which are $\mathbf{Q}$-linearly independent are algebraically independent.

There are several examples of numbers which can be written as the value of a polynomial at a point whose coordinates are logarithms of algebraic numbers. Among the most important ones are the determinants of square matrices whose entries are logarithms of algebraic numbers. Some regulators have this form; to decide whether they vanish or not may be considered as a transcendence problem. When they do not vanish, one expects them to be transcendental, and also not to be Liouville numbers.

7.2. Exponential periods. The next definition is from [38] $\S 4.3$. The remark after the introduction of [38] includes the sentence: "The last chapter, which is at a more advanced level and also more speculative than the rest of the paper, is by the first author only".

Definition An exponential period is an absolutely convergent integral of the product of an algebraic function with the exponent of an algebraic function, over a real semialgebraic set, where all polynomials entering the definition have algebraic coefficients. 
Exemple 44. In the algebra of exponential periods one finds of course all periods, as well as the numbers

$$
e^{\beta}=\int_{-\infty}^{\beta} e^{x} d x
$$

when $\beta$ is algebraic, the number

$$
\sqrt{\pi}=\int_{-\infty}^{\infty} e^{-x^{2}} d x
$$

the values of the Euler Gamma function at rational points

$$
\Gamma(s)=\int_{0}^{\infty} e^{-t} t^{s} \cdot \frac{d t}{t}
$$

as well as values of Bessel functions at algebraic points

$$
J_{n}(z)=\int_{|u|=1} \exp \left(\frac{z}{2}\left(u-\frac{1}{u}\right)\right) \frac{d u}{u^{n+1}} .
$$

These examples are viewed by S. Bloch and H. Esnault [11] as periods coming from a duality between homologic cycles and differential forms for connections with irregular singular points on Riemann surfaces. The referee pointed out to me that there are parallel works due to Masaaki Yoshida, Yoshishige Haraoka and Hironobu Kimura. I thank Cristiana Bertolin for providing me more information on this question.

7.3. Euler constant. No proof is available yet for the irrationality of Euler's constant

$$
\gamma=\lim _{n \rightarrow \infty}\left(1+\frac{1}{2}+\frac{1}{3}+\cdots+\frac{1}{n}-\log n\right)=0.5772157 \ldots
$$

(see [56]), even when a stronger assertion is expected, namely:

Conjecture 46. The number $\gamma$ is transcendental.

A still stronger statement is suggested in [38] $\S 1.1$ and $\S 4.3$ :

Conjecture 47. The number $\gamma$ is not a period, it is not even an exponential period.

7.4. A two dimensional analog of Euler constant. For each integer $k \geq 2$, denote by $A_{k}$ the minimal area of a closed disk in $\mathbf{R}^{2}$ containing at least $k$ points of $\mathbf{Z}^{2}$. For $n \geq 2$, set

$$
\delta_{n}=-\log n+\sum_{k=2}^{n} \frac{1}{A_{k}} \quad \text { and } \quad \delta=\lim _{n \rightarrow \infty} \delta_{n} .
$$


These quantities have been introduced and investigated by D.M. Masser in connection with Gel'fond's proof in 1929 of the transcendence of the number $e^{\pi}$, and then by F. Gramain [32] who conjectures:

\section{Conjecture 48 .}

$$
\delta=1+\frac{4}{\pi}\left(\gamma L^{\prime}(1)+L(1)\right)
$$

where $\gamma$ is Euler's constant (45) and

$$
L(s)=\sum_{n \geq 0}(-1)^{n}(2 n+1)^{-s} .
$$

is the L-function of the quadratic number field $\mathbf{Q}(i)$ (Dirichlet's Beta function).

Since $L(1)=\pi / 4$ and

$$
\begin{aligned}
L^{\prime}(1) & =\sum_{n \geq 0}(-1)^{n+1} \cdot \frac{\log (2 n+1)}{2 n+1} \\
& =\frac{\pi}{4}(3 \log \pi+2 \log 2+\gamma-4 \log \Gamma(1 / 4)),
\end{aligned}
$$

Conjecture 48 can be written

$$
\delta=1+3 \log \pi+2 \log 2+2 \gamma-4 \log \Gamma(1 / 4)=1.82282524 \ldots
$$

The best known estimate for $\delta$ is [32]

$$
1.811 \cdots<\delta<1.897 \ldots
$$

It seems likely that this number $\delta$ is not a period (hence that it is transcendental); however, given the lack of information we have on it, this number is probably not the best candidate to solve the question of [38] ( $\$ 1.2$ problem 3): exhibit at least one number which is not a period.

\section{Finite Characteristic}

Diophantine questions concerning real or complex numbers have analogs in function fields. In the case of finite characteristic a good reference is [31]. The first investigations go back to L. Carlitz (1935); the tools he introduced have been used in particular by I.I. Wade (1941) who proved the first transcendence statements in this context. After several works, in particular by J.M. Geijsel and P. Bundschuh in 1978, the theory has been developed in a deep way by Jing Yu after 1980, firstly in the situation of elliptic modules which were introduced by V.G. Drinfel'd in 1974, then, for the $t$-motives of G. Anderson after 1986. For a while, the results which were obtained in the situation of finite characteristic were analogues of the classical results related to complex numbers, until Jing $\mathrm{Yu}$ succeeded to prove new results which go much further than their complex counterparts [66]. 
The use, introduced in this context by L. Denis in 1990, of the derivative operator with respect to the variable of the function field, produces statements (like the transcendence of the derivative of the periods) which have no counterpart in the classical situation of complex numbers. Another peculiarity of finite characteristic is the possibility to use tensor products; they allow sometimes to reduce questions of algebraic independence to problems of linear independence - a nice example is given by S. David and L. Denis in [21]).

Two surveys on this subject give further information on this topic, the first one in 1992 by Jing Yu in [66], the other one in 1998 by W.D. Brownawell [13].

8.1. Euler constant in finite characteristic. A remarkable result in finite characteristic is the transcendence of the analog of Euler's constant. The complex number $\gamma$ (see (45)) can be defined as

$$
\gamma=\lim _{s \rightarrow 1}\left(\zeta(s)-\frac{1}{s-1}\right)
$$

where $\zeta$ denotes the Riemann zeta function

$$
\zeta(s)=\prod_{p}\left(1-p^{-s}\right)^{-1} .
$$

In this last product $p$ runs over the set of prime numbers. In finite characteristic the corresponding product is over the set of monic irreducible polynomials over a finite field $\mathbf{F}_{q}$ with $q$ elements. Here the product converges at the point $s=1$, and the value at this point is an analog of Euler's constant (it belongs to a completion $C$ of an algebraic closure of $\mathbf{F}_{q}((1 / T))$ ). This element of $C$ is transcendental over $\mathbf{F}_{q}(T)$ : this was proved by G.W. Anderson and D. Thakur in 1990 [4]. An interesting remark of them is that the tools which were available to I.I. Wade were already sufficient and the proof could have been given as early as in 1940 .

8.2. Thakur Gamma function. By analogy with the definition (11) of the Euler Gamma function as an infinite product, D. Thakur defines (cf. [13])

$$
\Gamma(z)=z^{-1} \prod_{n \in A_{+}}^{\infty}\left(1+\frac{z}{n}\right)^{-1},
$$

where $A=\mathbf{F}_{q}[T]$ is the ring of polynomials over the finite field $\mathbf{F}_{q}$ and where $A_{+}$is the set of monic polynomials. This Gamma function is meromorphic over the field $C$. It satisfies similar relations as the standard relations (20) which are satisfied by the Euler Gamma function. Also, in finite characteristic, the counterpart of the relations of Deligne-Koblitz-Ogus has been obtained by Deligne, Anderson and Thakur (see [14]). 
In 1992 G. Anderson introduced the notion of soliton, which, according to [14], is a higher dimensional version of the shtuka function for rank 1 Drinfeld modules. The meromorphic functions which were used by R. Coleman in his study of Frobenius endomorphisms on the curves of Fermat and Artin-Schreier had been interpreted by D. Thakur in terms of his Gamma function. While he was developing the parallel between these questions and the theory of partial differential equations, G. Anderson was led to introduce his solitons. This theory was used in 1997 by S.K. Sinha, who constructed $t$-modules whose periods have coordinates which are product of an algebraic number and values of Thakur Gamma function at rational points $a / f$, with $a$ and $f$ in $A_{+}$. By means of the earlier transcendence results of Jing Yu, S.K. Sinha could derive the transcendence of certain values of Thakur Gamma function at rational points.

These works were pursued by W.D. Brownawell and M.A. Papanikolas who show in [14] that the only linear relations with algebraic coefficients between the values of Thakur Gamma function are those which arise from Deligne-AndersonThakur relations. It is fair to say that this is the analog for function fields over a finite field of the Theorem of Wolfart and Wüstholz [64] on the linear independence of values of the Beta function.

A most remarkable fact is that it is possible to go further in the function field case. In [3], G.W. Anderson, W.D. Brownawell and M.A. Papanikolas show that all algebraic dependence relations between the values of Thakur Gamma function are consequences of the relations of Deligne-Anderson-Thakur.

For the values of the Gamma function, the situation in finite characteristic is much more advanced in the case of function fields than in the case of the complex field, where the Conjecture 22 of Rohrlich-Lang seems out of reach.

Very recently, M.A. Papanikolas succeeded to prove for Drinfeld modules the analog of the Conjecture 43 on algebraic independence of logarithms [46]. He develops a theory of Tannakian Galois groups for $t$-motives and relates this to the theory of Frobenius semilinear difference equations. He shows that the transcendence degree of the period matrix associated to a given $t$-motive is equal to the dimension of its Galois group. Using this result he proves that Carlitz logarithms of algebraic functions that are linearly independent over the rational function field are algebraically independent.

Another method (related to Mahler's method which was alluded to in $\S 7.1$ ) has been worked out by L. Denis, who also obtains results of algebraic independence of logarithms of algebraic points on Drinfeld modules.

\section{REFERENCES}

[1] B. AdAmczewski \& Y. Bugeaud - «On the complexity of algebraic numbers i. expansions in integer bases », Annals of Maths, 18pp., to appear. 
[2] B. Adamczewsiki, Y. Bugeaud \& F. Lucas - «Sur la complexité des nombres algébriques», C. R. Acad. Sci. Paris 339 (2004), p. 11-14.

[3] G. W. Anderson, W. D. Brownawell \& M. A. Papanikolas - «Determination of the algebraic relations among special Gamma-values in positive characteristic 》, Annals of Maths. 160 (2004), no. 1, p. 237-313, math.NT/0207168.

[4] G. W. Anderson \& D. S. Thakur - «Tensor powers of the Carlitz module and zeta values》, Ann. of Math. (2) 132 (1990), no. 1, p. 159-191.

[5] Y. ANDRÉ - G-functions and geometry, Aspects of Mathematics, E13, Friedr. Vieweg \& Sohn, Braunschweig, 1989.

[6] _ «Quelques conjectures de transcendance issues de la géométrie algébrique», Prépublication de l'institut de Mathématiques de Jussieu, 121, 1997.

[7] _ Une introduction aux motifs (motifs purs, motifs mixtes, périodes), Panoramas et Synthèses, vol. 17, Société Mathématique de France, Paris, 2004.

[8] K. Barré-Sirieix, G. Diaz, F. Gramain \& G. Philibert - «Une preuve de la conjecture de Mahler-Manin », Invent. Math. 124 (1996), no. 1-3, p. 1-9.

[9] C. Bertolin - «Périodes de 1-motifs et transcendance», J. Number Theory 97 (2002), no. 2, p. 204-221.

[10] F. Beukers \& J. Wolfart - «Algebraic values of hypergeometric functions》, in New advances in transcendence theory (Durham, 1986), Cambridge Univ. Press, 1988, p. 68-81.

[11] S. Bloch \& H. Esnault - «Gauss-Manin determinants for rank 1 irregular connections on curves», Math. Ann. 321 (2001), no. 1, p. 15-87.

[12] D. W. BoyD - «Mahler's measure and special values of $L$-functions - some conjectures 》, in Number theory in progress, Vol. 1 (Zakopane-Kościelisko, 1997), de Gruyter, Berlin, 1999, p. 27-34.

[13] W. D. Brownawell - «Transcendence in positive characteristic》, in Number theory (Tiruchirapalli, 1996), Contemp. Math., vol. 210, Amer. Math. Soc., Providence, RI, 1998, p. 317-332.

[14] W. D. Brownawell \& M. A. Papanikolas - «Linear independence of Gamma values in positive characteristic », J. reine angew. Math. 549 (2002), p. 91-148.

[15] S. BRultet - «D'une mesure d'approximation simultanée à une mesure d'irrationalité: le cas de $\Gamma(1 / 4)$ et $\Gamma(1 / 3) »$, Acta Arith. 104 (2002), no. 3, p. 243-281.

[16] Y. Bugeaud - Approximation by algebraic numbers, Cambridge Tracts in Math., vol. 160, Cambridge University Press, 2004.

[17] P. CARTIER - «Fonctions polylogarithmes, nombres polyzêtas et groupes pro-unipotents 》, Astérisque (2002), no. 282, p. Exp. No. 885, viii, 137-173, Séminaire Bourbaki, Vol. $2000 / 2001$.

[18] P. Cohen \& J. WOlfart - «Modular embeddings for some nonarithmetic Fuchsian groups》, Acta Arith. 56 (1990), no. 2, p. 93-110.

[19] P. B. Cohen \& G. Wüstholz - «Application of the André-Oort conjecture to some questions in transcendence», in A panorama of number theory or the view from Baker's garden (Zürich, 1999), Cambridge Univ. Press, Cambridge, 2002, p. 89-106.

[20] G. V. CUdnovs'KII - «Algebraic independence of constants connected with the exponential and the elliptic functions 》, Dokl. Akad. Nauk Ukrain. SSR Ser. A (1976), no. 8, p. 698-701, 767.

[21] S. David \& L. Denis - «Périodes de modules de Drinfel'd — "indépendance quadratique en rang II" », J. Ramanujan Math. Soc. 17 (2002), no. 1, p. 65-83.

[22] P. Deligne - «Cycles de Hodge absolus et périodes des intégrales des variétés abéliennes 》, Mém. Soc. Math. France (N.S.) (1980/81), no. 2, p. 23-33.

[23] P. Deligne \& A. B. Goncharov - «Groupes fondamentaux motiviques de Tate mixte», Ann. Sci. École Norm. Sup. (4) 38 (2005), no. 1, p. 1-56. 
[24] G. Diaz - «Utilisation de la conjugaison complexe dans l'étude de la transcendance de valeurs de la fonction exponentielle», J. Théor. Nombres Bordeaux (2004), p. 535-553.

[25] B. Edixhoven \& A. Yafaev - «Subvarieties of Shimura varieties》, Ann. of Math. (2) 157 (2003), no. 2, p. 621-645.

[26] N. I. FEL'DMAN \& Y. V. NESTERENKo - «Transcendental numbers》, in Number theory, IV (I. R. Shafarevich, éd.), Encyclopaedia Math. Sci., vol. 44, Springer, Berlin, 1998, Transcendental numbers, A translation of Number theory. 4 (Russian), Ross. Akad. Nauk, Vseross. Inst. Nauchn. i Tekhn. Inform., Moscow, Translation by N. Koblitz, Translation edited by A. N. Parshin and I. R. Shafarevich, p. vi+345.

[27] S. Fischler - «Irrationalité de valeurs de zêta (d'après Apéry, Rivoal, ... )», Astérisque (2004), no. 294, p. 27-62, Sém. Bourbaki 2002/03, exp. no. 910.

[28] É. GAUdRON - «Mesure d'indépendance linéaire de logarithmes dans un groupe algébrique commutatif», C. R. Acad. Sci. Paris Sér. I Math. 333 (2001), no. 12, p. 1059-1064.

[29] _ _ «Mesures d'indépendance linéaire de logarithmes dans un groupe algébrique commutatif», Invent. Math. 162 (2005), no. 1, p. 137-188.

[30] A. B. Goncharov - «Multiple $\zeta$-values, Galois groups, and geometry of modular varieties》, in European Congress of Mathematics, Vol. I (Barcelona, 2000), Progr. Math., vol. 201, Birkhäuser, Basel, 2001, p. 361-392.

[31] D. Goss, D. R. HAyes \& M. I. Rosen (éds.) - The arithmetic of function fields, Ohio State University Mathematical Research Institute Publications, 2, Berlin, Walter de Gruyter \& Co., 1992.

[32] F. GRAmain \& M. WEBER - «Computing an arithmetic constant related to the ring of gaussian integers 》, Math. Comp. 44 (1985), no. 169, p. 241-250 and S13-S16, Corrigendum, id. 48 (1987) no. 178 p. 854.

[33] P. GRINSPAN - «Measures of simultaneous approximation for quasi-periods of abelian varieties 》, J. Number Theory 94 (2002), no. 1, p. 136-176.

[34] B. H. Gross - «On the periods of abelian integrals and a formula of Chowla and Selberg », Invent. Math. 45 (1978), no. 2, p. 193-211.

[35] A. Grothendieck - «On the de Rham cohomology of algebraic varieties 》, Inst. Hautes Études Sci. Publ. Math. (1966), no. 29, p. 95-103.

[36] M. E. Hoffman - «The algebra of multiple harmonic series》, J. Algebra 194 (1997), no. 2, p. $477-495$.

[37] N. Koblitz \& D. Rohrlich - «Simple factors in the Jacobian of a Fermat curve», Canad. J. Math. 30 (1978), no. 6, p. 1183-1205.

[38] M. Kontsevich \& D. ZagieR - «Periods》, in Mathematics unlimited-2001 and beyond, Springer, Berlin, 2001, p. 771-808.

[39] S. LANG - Introduction to transcendental numbers, Addison-Wesley Publishing Co., Reading, Mass.-London-Don Mills, Ont., 1966, =Collected papers. Vol. II (1952-1970), SpringerVerlag 2000, p. 396-506.

[40] _ _ «Relations de distributions et exemples classiques》, in Séminaire Delange-PisotPoitou, 19e année: 1977/78, Théorie des nombres, Fasc. 2, Secrétariat Math., Paris, 1978, =Collected papers. Vol. III (1978-1990), Springer-Verlag 2000, p. 59-65, p. Exp. No. 40, 6 .

[41] D. MASSER - «Interpolation on group varieties》, in Approximations diophantiennes et nombres transcendants (Luminy, 1982) (D. Bertrand \& M. Waldschmidt, éds.), Progress in Math., no. 31, Birkhäuser, 1983, p. 151-171.

[42] Y. V. Nesterenko - «Modular functions and transcendence questions», Mat. Sb. [Sb. Math] 187 (1996), no. 9, p. 65-96 [1319-1348].

[43] Y. V. Nesterenko \& P. PhiLippon (éds.) - Introduction to algebraic independence theory, Lecture Notes in Math., no. 1752, Springer, 2001. 
[44] K. NishiokA - Mahler functions and transcendence, Lecture Notes in Mathematics, vol. 1631, Springer-Verlag, Berlin, 1996.

[45] F. OorT - «Canonical liftings and dense sets of CM-points》, in Arithmetic geometry (Cortona, 1994), Sympos. Math., XXXVII, Cambridge Univ. Press, Cambridge, 1997, p. 228234.

[46] M. A. Papanikolas - «Tannakian duality for Anderson-Drinfel'd motives and algebraic independence of Carlitz logarithms 》, http://arxiv.org/abs/math/0506078, 2005.

[47] F. Pellarin - «Idéaux stables dans certains anneaux différentiels de formes quasimodulaires de Hilbert 》, math.NT/0407381, 2004.

[48] A. J. VAN DER POORTEN - «On the arithmetic nature of definite integrals of rational functions. 》, Proc. Amer. Math. Soc. 29 (1971), p. 451-456.

[49] G. Rhin \& C. Viola - «The group structure for $\zeta(3) »$, Acta Arith. 97 (2001), no. 3, p. 269-293.

[50] T. SCHnEIDER - «Transzendenzuntersuchungen periodischer Funktionen》, J. reine angew. Math. 14 (1934), p. 65-74.

[51] _ «Arithmetische Untersuchungen elliptischer Integrale», Math. Ann. 113 (1937), p. $1-13$.

[52] _ «Zur Theorie des Abelschen Funktionen und Integrale», J. reine angew. Math. 183 (1941), p. 110-128.

[53] T. SchneIDER - Einführung in die transzendenten Zahlen, Springer-Verlag, Berlin, 1957, trad. fr.: Introduction aux nombres transcendants, Gauthier-Villars Paris, 1959.

[54] D. Shanks - «Incredible identities》, Fibonacci Quart. 12 (1974), p. 271, 280.

[55] C. L. Siegel - Transcendental Numbers, Annals of Mathematics Studies, no. 16, Princeton University Press, Princeton, N. J., 1949.

[56] J. Sondow - «Criteria for irrationality of Euler's constant», Proc. Amer. Math. Soc. 131 (2003), no. 11, p. 3335-3344.

[57] T. Terasoma - «Mixed Tate motives and multiple zeta values », Invent. Math. 149 (2002), no. 2, p. 339-369.

[58] K. G. VASIL'EV - «On the algebraic independence of the periods of Abelian integrals», Mat. Zametki 60 (1996), no. 5, p. 681-691, 799.

[59] M. WALDSChMIDT - Diophantine approximation on linear algebraic groups, Grundlehren der Mathematischen Wissenschaften [Fundamental Principles of Mathematical Sciences], vol. 326, Springer-Verlag, Berlin, 2000.

[60] _ _ «Open diophantine problems», Moscow Mathematical Journal 4 (2004), p. 245305.

[61] _ «Variations on the six exponentials theorem》, in Algebra and Number Theory, Hindustan Book Agency, 2005, with an appendix by H. Shiga: Periods of the Kummer surface, p. 338-358.

[62] J. Wolfart - «Werte hypergeometrischer Funktionen》, Invent. Math. 92 (1988), no. 1, p. $187-216$.

[63] - «Values of Gauss' continued fractions 》, in Number theory, Vol. II (Budapest, 1987), Colloq. Math. Soc. János Bolyai, vol. 51, North-Holland, Amsterdam, 1990, p. 1051-1063.

[64] J. Wolfart \& G. Wüstholz - «Der Überlagerungsradius gewisser algebraischer Kurven und die Werte der Betafunktion an rationalen Stellen», Math. Ann. 273 (1985), no. 1, p. 1-15.

[65] G. WÜstholz - «Algebraische Punkte auf analytischen Untergruppen algebraischer Gruppen», Ann. of Math. (2) $\mathbf{1 2 9}$ (1989), no. 3, p. 501-517.

[66] J. YU - «Transcendence in finite characteristic», in The arithmetic of function fields (Columbus, OH, 1991), Ohio State Univ. Math. Res. Inst. Publ., vol. 2, de Gruyter, Berlin, 1992, p. 253-264. 
Michel Waldschmidt

Institut de Mathématiques de Jussieu - UMR 7586 du CNRS, Université P. et M. Curie (Paris VI)

175 rue du Chevaleret, F-75013 Paris

E-mail:miw@math.jussieu.fr

http://www.math.jussieu.fr/ miw 